\title{
Evaluación semicuantitativa de gliomas cerebrales en adultos: un enfoque de las características neuropatológicas
}

Marco Antonio Rodríguez-Florido, ${ }^{1}$ Iris A. Feria-Romero, ${ }^{1}$ Bárbara Nettel-Rueda, ${ }^{2}$ José Guerrero-Cantera, ${ }^{1}$ Sandra Orozco-Suárez, ${ }^{1}$ Jesús A. Chavez, ${ }^{1}$ Gerardo Guinto ${ }^{2}$ e Israel Grijalva ${ }^{1 *}$ ${ }^{1}$ Unidad de Investigación Médica en Enfermedades Neurológicas; ${ }^{2}$ Hospital de Especialidades, Servicio de Neurocirugía. Instituto Mexicano del Seguro Social, Centro Médico Nacional Siglo XXI, Ciudad de México, México

\section{Resumen}

Introducción: Los gliomas son neoplasias con alta recurrencia y mortalidad. Por la dificultad para aplicar la clasificación de la Organización Mundial de la Salud (2016), los países en desarrollo siguen utilizando la evaluación histológica para diagnosticarlos y clasificarlos. Objetivo: Desarrollar una escala semicuantitativa para calificar numéricamente las características morfológicas de los gliomas. Método: Cohorte de pacientes con gliomas evaluada y seguida durante 36 meses. Se analizaron y calificaron cortes del tejido tumoral, incluyendo aspectos como estirpe celular, celularidad, pleomorfismo nuclear, mitosis, hiperplasia endotelial, cambios hipóxicos, cuerpos apoptóticos, necrosis, hemorragia e índice de proliferación. Resultados: Se analizaron 58 casos. La mediana de la calificación de los gliomas de bajo grado fue de 12 puntos (percentiles 25 y 75 de 9 y 13.5, respectivamente) y la de los gliomas de alto grado fue de 17 puntos (percentiles 25 y 75 de 16 y 20.5, respectivamente) ( $p$ < 0.0001). La supervivencia a 36 meses de los pacientes con gliomas de bajo (13/17) y alto grado (6/41) también fue significativamente diferente $(p<0.0001)$. Conclusiones: La escala morfológica semicuantitativa permite una evaluación objetiva de los gliomas, con una adecuada correlación entre la calificación, el grado del tumor y el tiempo de supervivencia.

PALABRAS CLAVE: Glioblastoma. Glioma de bajo grado. Graduación semicuantitativa. Evaluación neuropatológica.

\begin{abstract}
Introduction: Gliomas are neoplasms with high recurrence and mortality. Due to the difficulty to apply the World Health Organization (2016) classification, developing countries continue to use histological evaluation to diagnose and classify these neoplasms. Objective: To develop a semi-quantitative scale to numerically grade gliomas morphological characteristics. Method: A cohort of patients with gliomas was assessed and followed for 36 months. Tumor tissue sections were analyzed and graded, including aspects such as cell line, cellularity, nuclear pleomorphism, mitosis, endothelial hyperplasia, hypoxic changes, apoptotic bodies, necrosis, hemorrhage and proliferation index. Results: 58 cases were analyzed. Low-grade gliomas median score was 12 points ( 9 and 13.5 for percentiles 25 and 75, respectively), whereas for high-grade gliomas it was 17 points (16 and 20.5 for percentiles 25 and 75 , respectively) $(p<0.0001)$. Thirty-six-month survival of patients with low (13/17) and high grade gliomas $(6 / 41)$ was also significantly different $(p<0.0001)$. Conclusions: The semi-quantitative morphological scale allows an objective evaluation of gliomas, with an adequate correlation between the score, tumor grade and survival time.
\end{abstract}

KEY WORDS: Glioblastoma. Low-grade glioma. Semi-quantitative grading. Neuropathological assessment.

Correspondencia:

*Israel Grijalva

E-mail: igrijalvao@yahoo.com
Fecha de recepción: 09-11-2018

Fecha de aceptación: 19-03-2019

DOI: 10.24875/GMM.19004832
Gac Med Mex. 2019;155:473-480

Disponible en PubMed

www.gacetamedicademexico.com 


\section{Introducción}

Los gliomas son tumores cerebrales primarios cuyas células precursoras muestran morfología y expresión génica similares a las del tejido glial. Entre ellos se incluyen astrocitomas, oligodendrogliomas y ependimomas. La clasificación actualizada de los gliomas humanos se basa en los criterios histológicos emitidos por la Organización Mundial de la Salud y marcadores moleculares específicos. ${ }^{1}$ El grado se asigna de I a IV en función de características morfológicas como proliferación vascular, mitosis, pleomorfismo y necrosis, entre otras. Sin embargo, estos criterios dejan espacio a la interpretación subjetiva y dan lugar a la variabilidad intra e interobservador entre los neuropatólogos. La clasificación errónea del glioma en la evaluación histológica inicial impide la determinación precisa de su incidencia y prevalencia y puede tener un impacto negativo en la atención del paciente. ${ }^{2}$

En la actualidad, los marcadores moleculares son parte de los criterios diagnósticos debido a que la reciente clasificación de la Organización Mundial de la Salud, en el apartado de tumores del sistema nervioso central, incluye la codeleción 1p/19q (LOH), la mutación de la proteína IDH1 y la mutación del promotor del gen ATRX, como parte de la evaluación obligatoria de los gliomas, especialmente en los centros de atención a la salud donde el diagnóstico de oligoastrocitomas es frecuente. ${ }^{1}$ Desafortunadamente, las nuevas técnicas de genotipificación o ensayos inmunohistoquímicos no siempre están disponibles en los países en desarrollo; por esta razón, la clasificación actualizada ha incluido la categoría "designación no específica" cuando no se cuenta con herramientas adecuadas para la descripción de la estirpe celular. ${ }^{1}$ Por lo anterior, y reconociendo esas limitaciones, es importante mejorar los criterios morfológicos que ayuden a determinar grado histológico, tamaño, ubicación y patrones de diferenciación celular, que además de mejorar la precisión diagnóstica contribuyan al establecimiento del pronóstico. ${ }^{3}$

En la investigación que se presenta se propuso el uso de una escala semicuantitativa basada en características morfológicas que incluyen otras no utilizadas actualmente para la clasificación histológica como hiperplasia endotelial, cuerpos apoptóticos, hemorragia y cambios hipóxicos; se pretende que con el uso de esta herramienta se mejore la variabilidad interobservador, el diagnóstico y la clasificación de estos tumores y sirva como un primer paso para el pronóstico.

\section{Método}

Cohorte de pacientes con diagnóstico presuntivo de glioma, atendidos en el Departamento de Neurocirugía del Hospital de Especialidades, Centro Médico Nacional Siglo XXI, quienes fueron reclutados entre 2011 y 2014 y que por escrito aceptaron participar, previa información relativa a la investigación. El presente protocolo fue aprobado por el Comité Nacional de Investigación Científica del Instituto Mexicano del Seguro Social. Los criterios de inclusión abarcaron casos con diagnóstico presuntivo inicial de glioma, confirmado por dos neuropatólogos expertos. Ante discrepancia diagnóstica, se solicitó la evaluación de un tercer neuropatólogo. Cuando dos diagnósticos neuropatológicos concordaron, se estableció el diagnóstico final. Para determinar el tiempo de supervivencia, todos los pacientes fueron seguidos al menos 36 meses después de la cirugía.

Las muestras de tejido, fijadas en formalina e incluidas en parafina, se obtuvieron del archivo del Departamento de Patología del hospital. La morfología del tumor se determinó en cortes de tejido de $5 \mu \mathrm{m}$, teñidas con hematoxilina y eosina. La detección inmunohistoquímica de Ki67 se realizó con el reactivo DIVA $^{\mathrm{TM}}$, el anticuerpo primario Ki67 (ambos de Biocare Medical, Concord, California, Estados Unidos) y el sistema de detección ImmPRESS ${ }^{\text {TM }}$ HRP Universal Antibody (IgG antirratón/lgG anticonejo, peroxidasa; Vector Lab, Estados Unidos). Finalmente, el anticuerpo secundario se reveló con diaminobencidina (Biocare Medical, Concord, California, Estados Unidos). Todas las laminillas se visualizaron con un microscopio óptico (modelo E600, Nikon Eclipse, Japón).

\section{Evaluación semicuantitativa de los gliomas}

La evaluación del tejido tumoral se realizó con las laminillas disponibles para cada caso, dependiendo del tamaño del tumor, conforme las siguientes características: estirpe celular, celularidad, pleomorfismo nuclear, mitosis, hiperplasia endotelial, cambios hipóxicos, cuerpos apoptóticos, necrosis, hemorragia e índice de proliferación. De acuerdo con la estirpe celular, los tumores se dividieron en astrocitoma, oligodendroglioma, ependimoma o "designación no específica", anteriormente identificados como oligoastrocitomas, gangliogliomas o gliomas mixtos. ${ }^{4} \mathrm{~A}$ las neoplasias astrocíticas se les asignó una puntuación de 3 (calificación alta), debido a su 
Tabla 1. Evaluación morfológica semicuantitativa de gliomas cerebrales*

\begin{tabular}{|c|c|c|c|c|c|c|}
\hline \multicolumn{2}{|l|}{ Parámetro } & \multicolumn{4}{|c|}{ Descripción de escala y puntuación } & \multirow[t]{2}{*}{ Puntuación } \\
\hline Estirpe celular & $\begin{array}{l}\text { Tipo } \\
\text { Puntuación }\end{array}$ & $\begin{array}{l}\text { Ependimal } \\
0\end{array}$ & $\begin{array}{c}\text { Oligodendrocito } \\
1\end{array}$ & $\begin{array}{c}\text { Designación no específica } \\
2\end{array}$ & $\begin{array}{c}\text { Astrocito } \\
3\end{array}$ & \\
\hline $\begin{array}{l}\text { Celularidad } \\
\text { Evaluación a 10x }\end{array}$ & $\begin{array}{l}\text { Porcentaje } \\
\text { Puntuación }\end{array}$ & $\begin{array}{c}<10 \% \\
1\end{array}$ & $\begin{array}{c}11-25 \% \\
2\end{array}$ & $\begin{array}{c}>25 \% \\
3\end{array}$ & & \\
\hline Evaluación a 40x & $\begin{array}{l}\text { Leve/moderado/ } \\
\text { severo } \\
\text { Puntuación }\end{array}$ & $\begin{array}{l}\text { Núcleos regulares } \\
\text { y monótonos, } \\
\text { tamaño de } \\
7-10 \mu \mathrm{m} \\
1\end{array}$ & $\begin{array}{c}\text { Núcleos con } \\
\text { tamaño variable de } \\
10-15 \mu \mathrm{m} \text { y cromatina } \\
\text { compacta } \\
2\end{array}$ & $\begin{array}{l}\text { Núcleos de tamaño } \\
\text { irregular }>15 \mu \mathrm{m} \text { con } \\
\text { cromatina granular y } \\
\text { abierta } \\
3\end{array}$ & & \\
\hline $\begin{array}{l}\text { Mitosis } \\
\text { Diez campos a } 40 x\end{array}$ & $\begin{array}{l}\text { Número } \\
\text { Puntuación }\end{array}$ & $\begin{array}{c}<5 \\
1\end{array}$ & $\begin{array}{c}6-10 \\
2\end{array}$ & $\begin{array}{c}>10 \\
3\end{array}$ & & \\
\hline $\begin{array}{l}\text { Hiperplasia endotelial } \\
\text { (Vasos sanguíneos) } \\
\text { Evaluación a 10x }\end{array}$ & $\begin{array}{l}\text { Número } \\
\text { Puntuación }\end{array}$ & $\begin{array}{l}0 \\
0\end{array}$ & $\begin{array}{c}1-5 \\
1\end{array}$ & $\begin{array}{c}6-10 \\
2\end{array}$ & $\begin{array}{c}>10 \\
3\end{array}$ & \\
\hline $\begin{array}{l}\text { Hipoxia } \\
\text { Diez campos a 40x }\end{array}$ & $\begin{array}{l}\text { Número } \\
\text { Puntuación }\end{array}$ & $\begin{array}{l}0 \\
0\end{array}$ & $\begin{array}{c}1-5 \\
1\end{array}$ & $\begin{array}{c}6-10 \\
2\end{array}$ & $\begin{array}{c}>10 \\
3\end{array}$ & \\
\hline $\begin{array}{l}\text { Apoptosis } \\
\text { Cuerpos apoptóticos } \\
\text { Diez campos a } 40 x\end{array}$ & $\begin{array}{l}\text { Número } \\
\text { Puntuación }\end{array}$ & $\begin{array}{l}0 \\
0\end{array}$ & $\begin{array}{c}1-5 \\
1\end{array}$ & $\begin{array}{c}6-10 \\
2\end{array}$ & $\begin{array}{c}>10 \\
3\end{array}$ & \\
\hline $\begin{array}{l}\text { Necrosis } \\
\text { Evaluación a 10x }\end{array}$ & $\begin{array}{l}\text { Porcentaje } \\
\text { Puntuación }\end{array}$ & $\begin{array}{c}0 \% \\
0\end{array}$ & $\begin{array}{c}1 \mathrm{a}<30 \% \\
1\end{array}$ & $\begin{array}{c}30 \mathrm{a}<70 \% \\
2\end{array}$ & $\begin{array}{c}>70 \% \\
3\end{array}$ & \\
\hline $\begin{array}{l}\text { Hemorragia } \\
\text { Corte del tejido a } 10 x\end{array}$ & $\begin{array}{l}\text { Porcentaje } \\
\text { Puntuación }\end{array}$ & $\begin{array}{c}0 \% \\
0\end{array}$ & $\begin{array}{c}1 \mathrm{a}<30 \% \\
1\end{array}$ & $\begin{array}{c}30 a<70 \% \\
2\end{array}$ & $\begin{array}{c}>70 \% \\
3\end{array}$ & \\
\hline $\begin{array}{l}\text { Índice de proliferación } \\
\text { Expresión nuclear de } \\
\text { Ki67 } \\
\text { Evaluación a 40x }\end{array}$ & $\begin{array}{l}\text { Porcentaje } \\
\text { Puntuación }\end{array}$ & $0 \%$ & $1 \mathrm{a}<10 \%$ & $10 a<20 \%$ & $\begin{array}{c}>20 \% \\
3\end{array}$ & \\
\hline \multicolumn{7}{|l|}{ Puntuación total } \\
\hline $\begin{array}{l}\text { La evaluación se realizó en toda } \\
\text { zampos a 40x: diez diferentes ce } \\
\text { elación con su malla fibrilar (MF } \\
\text { tivisión por campo a 40x. La hip } \\
\text { zon picnosis. La necrosis y la he }\end{array}$ & $\begin{array}{l}\text { ). El pleomorfismo cor } \\
\text { perplasia endotelial se } \\
\text { emorragia se determin }\end{array}$ & les para cada caso. Evaluac & $\begin{array}{l}\text { ad de los núcleos y la condens } \\
\text { asos por campo. Los cambios } \\
\text { tes de tejido por muestra. El ín }\end{array}$ & $\begin{array}{l}\text { aso completo es evaluado con un aum } \\
\text { mina por el porcentaje de células (\% } \\
\text { ación de la cromatina. La mitosis cuant } \\
\text { hipóxicos se identifican por células nec } \\
\text { dice de proliferación se determina por }\end{array}$ & $\begin{array}{l}\text { ento de } 10 \times 0 \\
\text { lular), que se } \\
\text { ica el número } \\
\text { olásicas hipere } \\
\text { porcentaje de }\end{array}$ & $\begin{array}{l}\text { 40x. Diez } \\
\text { observa en } \\
\text { de células en } \\
\text { eosinofilicas } \\
\text { e células con }\end{array}$ \\
\hline
\end{tabular}

naturaleza difusamente infiltrante y a su alta recurrencia secundaria a la dificultad de una resección completa. A las neoplasias "designación no específica" se les asignó una puntuación de 2 (intermedio), debido a su estirpe celular astrocítica mixta; las otras neoplasias de estirpe celular como los oligodendrogliomas y ependimomas recibieron una puntuación de 1 y 0 (bajo), respectivamente, debido a su comportamiento benigno (Tabla 1).

Las siguientes variables se evaluaron de acuerdo con las características que presentaban y se les asignó un valor numérico entre 0 y 3 : 0 ausencia, 1 escaso, 2 moderado y 3 abundante (Tabla 1). La celularidad se determinó de acuerdo con el porcentaje de células en relación con su malla fibrilar (MF, célula:MF) con un aumento de 10x. El pleomorfismo nuclear se evaluó en función del tamaño y la regularidad de los núcleos, así como de la condensación de la cromatina con un aumento de 40x, dando un valor de 1 a los núcleos regulares y monótonos con tamaño entre 7 y $10 \mu \mathrm{m}$. El número de mitosis se calculó por campo, considerando 10 campos a un aumento de 40x. La hiperplasia endotelial se calculó por el número de vasos por campo con un aumento de 10x. Los cambios hipóxicos fueron identificados por células neoplásicas hipereosinofílicas con picnosis, cuantificando 10 campos con un aumento de 40x. La necrosis y la hemorragia se determinaron mediante porcentaje por campo en cada muestra con un aumento de 10x. Finalmente, el índice de proliferación se determinó por el porcentaje de expresión nuclear de Ki67 con un aumento de $40 x$. 
Tabla 2. Datos demográficos, localización, lateralidad y diagnóstico de los pacientes con gliomas de bajo y alto grado

\begin{tabular}{|c|c|c|c|c|c|c|c|c|c|}
\hline Variables & \multicolumn{3}{|r|}{ Total } & \multicolumn{3}{|c|}{ Bajo-grado } & \multicolumn{3}{|c|}{ Alto-grado } \\
\hline & \multicolumn{3}{|c|}{$52.1 \pm 16.4$} & \multicolumn{3}{|c|}{$44.4 \pm 16.9$} & \multicolumn{3}{|c|}{$55.3 \pm 15.2$} \\
\hline $\begin{array}{l}\text { Datos demográficos } \\
\text { Edad, años } \pm \text { DE } \\
\text { Pacientes } \\
\text { Hombres }\end{array}$ & $\begin{array}{l}n \\
58 \\
36\end{array}$ & $\begin{array}{c}\% \\
100 \\
60.3\end{array}$ & $\begin{array}{c}\text { Izquierdo/derecho } \\
- \\
-\end{array}$ & $\begin{array}{c}n \\
17 \\
13\end{array}$ & $\begin{array}{c}\% \\
29.3 \\
65.0\end{array}$ & $\begin{array}{c}\text { Izquierdo/derecho } \\
- \\
-\end{array}$ & $\begin{array}{l}n \\
41 \\
23\end{array}$ & $\begin{array}{c}\% \\
70.7 \\
56.1\end{array}$ & $\begin{array}{c}\text { Izquierdo/derecho } \\
- \\
-\end{array}$ \\
\hline $\begin{array}{l}\text { Lóbulos afectados } \\
1 \\
2 \\
3 \\
\text { Extralobar }\end{array}$ & $\begin{array}{l}35 \\
19 \\
1 \\
3\end{array}$ & $\begin{array}{c}60.3 \\
32.7 \\
1.7 \\
5.2\end{array}$ & $\begin{array}{c}18 / 17 \\
8 / 11 \\
0 / 1 \\
-\end{array}$ & $\begin{array}{l}13 \\
2 \\
- \\
2\end{array}$ & $\begin{array}{l}76.5 \\
11.8 \\
11.7\end{array}$ & $\begin{array}{l}8 / 5 \\
0 / 2 \\
- \\
-\end{array}$ & $\begin{array}{c}22 \\
17 \\
1 \\
1\end{array}$ & $\begin{array}{l}53.7 \\
41.5 \\
2.4 \\
2.4\end{array}$ & $\begin{array}{l}10 / 12 \\
8 / 9 \\
0 / 1 \\
-\end{array}$ \\
\hline $\begin{array}{l}\text { Localización } \\
\text { Frontal } \\
\text { Parietal } \\
\text { Temporal } \\
\text { Occipital }\end{array}$ & $\begin{array}{l}29 \\
22 \\
16 \\
9\end{array}$ & $\begin{array}{l}38.2 \\
28.9 \\
21.1 \\
11.8\end{array}$ & $\begin{array}{l}16 / 13 \\
7 / 15 \\
5 / 11 \\
5 / 4\end{array}$ & $\begin{array}{l}10 \\
2 \\
4 \\
1\end{array}$ & $\begin{array}{c}58.8 \\
11.8 \\
23.5 \\
5.9\end{array}$ & $\begin{array}{l}6 / 4 \\
0 / 2 \\
2 / 2 \\
0 / 1\end{array}$ & $\begin{array}{c}19 \\
20 \\
12 \\
8\end{array}$ & $\begin{array}{l}32.2 \\
33.9 \\
20.3 \\
13.6\end{array}$ & $\begin{array}{l}10 / 9 \\
7 / 13 \\
3 / 9 \\
5 / 3\end{array}$ \\
\hline $\begin{array}{l}\text { Diagnóstico histopatológico } \\
\text { Glioblastoma } \\
\text { A III } \\
\text { DNE III } \\
\text { E III } \\
\text { A II } \\
\text { DNE II } \\
\text { O II } \\
\text { E II } \\
\text { Total }\end{array}$ & $\begin{array}{c}38 \\
1 \\
1 \\
1 \\
10 \\
5 \\
1 \\
1 \\
58\end{array}$ & $\begin{array}{c}65.5 \\
1.7 \\
1.7 \\
1.7 \\
17.2 \\
8.6 \\
1.7 \\
1.7 \\
100\end{array}$ & $\begin{array}{c}16 / 22 \\
0 / 1 \\
0 / 1 \\
1 / 0 \\
3 / 7 \\
4 / 1 \\
1 / 0 \\
0 / 1 \\
25 / 33\end{array}$ & $\begin{array}{c}- \\
- \\
- \\
- \\
10 \\
5 \\
1 \\
1 \\
17\end{array}$ & $\begin{array}{c}- \\
- \\
- \\
- \\
58.8 \\
29.4 \\
5.9 \\
5.9 \\
100\end{array}$ & $\begin{array}{l}- \\
- \\
- \\
- \\
3 / 7 \\
4 / 1 \\
1 / 0 \\
0 / 1 \\
8 / 9\end{array}$ & $\begin{array}{c}38 \\
1 \\
1 \\
1 \\
- \\
- \\
- \\
- \\
41\end{array}$ & $\begin{array}{c}95.0 \\
2.4 \\
2.4 \\
2.4 \\
- \\
- \\
- \\
- \\
100\end{array}$ & $\begin{array}{c}16 / 22 \\
0 / 1 \\
0 / 1 \\
1 / 0 \\
- \\
- \\
- \\
- \\
17 / 24\end{array}$ \\
\hline
\end{tabular}

A III = astrocitoma anaplásico grado III, DNE III = designación no específica grado III, E III = ependimoma anaplásico grado III, A II = xantoastrocitoma pleomórfico, astrocitoma difuso astrocitoma gemistocístico; DNE II = designación no específica grado II, O II = oligodendroglioma grado II, E II = ependimoma grado II.

\section{Análisis estadístico}

El análisis descriptivo incluyó frecuencia, porcentajes, promedio \pm desviación estándar (DE) o mediana con percentiles 25 y 75 (p25-p75) de acuerdo con su distribución. Las diferencias entre las puntuaciones histopatológicas de los grupos tumorales de bajo y alto grado se estimaron utilizando la prueba no paramétrica de $U$ de Mann-Whitney, y la supervivencia global con la prueba exacta de Fisher, calculada con GraphPad Prism versión 5.0. Un valor de $p<0.05$ se consideró estadísticamente significativo.

\section{Resultados}

Fueron evaluados 58 pacientes, con una edad promedio de $52.1 \pm 16.4$ años; predominó el sexo masculino (60.3\%). Los neuropatólogos coincidieron en 48 casos (83\%). Un tercer neuropatólogo evaluó 10 casos discordantes; una vez que coincidió la opinión de dos evaluadores, se estableció el diagnóstico final. La localización más frecuente del tumor fue el hemisferio izquierdo (56.6\%), con predominio en los lóbulos frontal y parietal (38.2 y $28.9 \%$, respectivamente); $84 \%$ de los tumores fue de origen astrocítico (49/58), de los cuales el glioblastoma fue el más común (65.5\%). Los tumores se dividieron en bajo (grados I y II) y alto grado (grados III y IV). La edad media de los pacientes con gliomas de bajo grado $(n=17)$ fue de $44.4 \pm 17$ años y de $55.3 \pm$ 15 años para aquellos con gliomas de alto grado $(n=41)$. Se observó predominio del sexo masculino en ambos grupos: 65 y $56.1 \%$, respectivamente (Tabla 2).

Las características morfológicas de los tumores incluidos en la evaluación semicuantitativa se muestran en la Figura 1. El rango de calificación de los tumores fue de 6 a 26 puntos (incluyendo cada parámetro). La mediana para los gliomas de bajo grado fue de 12 puntos (9-13.5) y de 17 puntos (16-20.5) para los gliomas de alto grado, lo que mostró una diferencia estadística significativa $(p<0.0001)$.

Se estimaron las puntuaciones de las principales características biológicas de los astrocitomas malignos, como la proliferación celular, la angiogénesis y la muerte celular. El valor de proliferación celular se obtuvo sumando las puntuaciones de los siguientes parámetros: celularidad, pleomorfismo nuclear, mitosis y Ki67, observando diferencia significativa entre ambos grupos $(p=0.0002)$. El valor de la angiogénesis se 

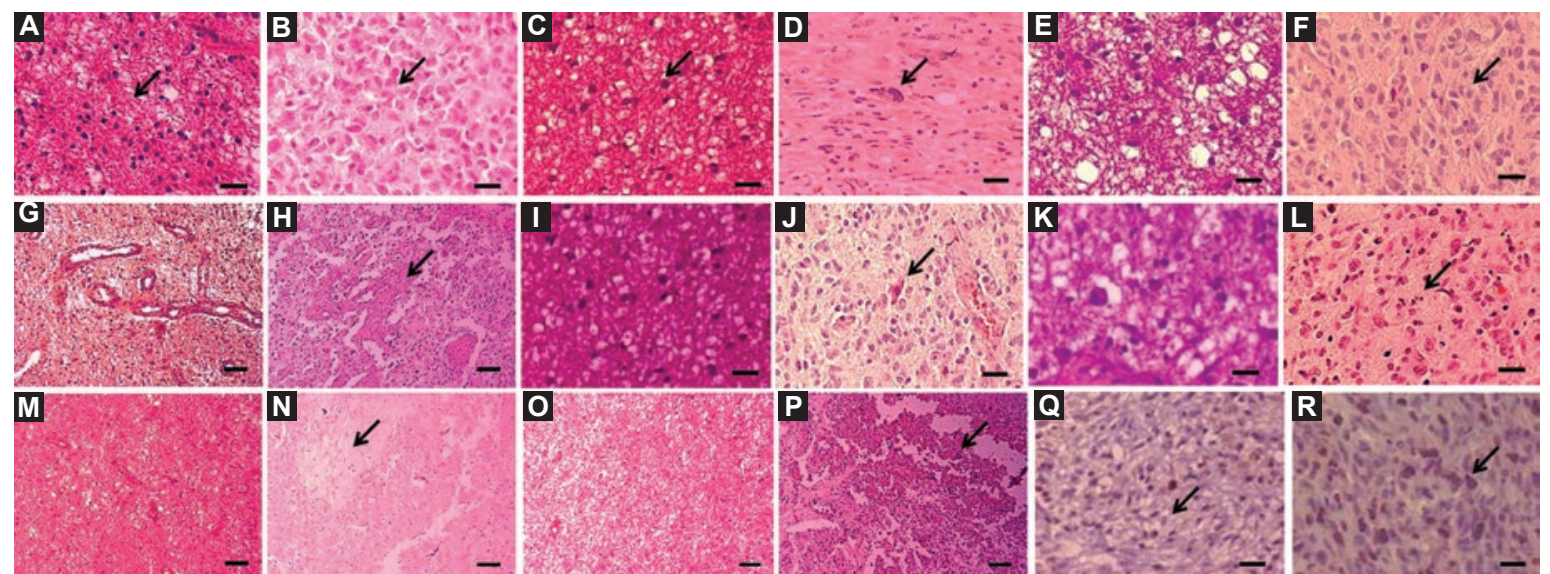

Figura 1. Características celulares para la evaluación morfológica semicuantitativa de gliomas cerebrales. Las puntuaciones corresponden a las características indicadas en la Tabla 1. Celularidad: A) Puntuación de 2 y porcentaje celular de 15 \%; B) Puntuación de 3 y porcentaje celular de 50 \%. Pleomorfismo nuclear: C) Puntuación de 1 (núcleos regulares, 7 a $10 \mu \mathrm{m}$ ); D) Puntuación de 3 (núcleos irregulares, $>15 \mu \mathrm{m}$ ). Mitosis: E) Puntuación de 1 (0-5 mitosis); F) Puntuación de 3 (> 10 mitosis). Hiperplasia endotelial: G) Puntuación de 0 (ausente); H) Puntuación de 3 (> 10 vasos sanguíneos). Hipoxia: I) Puntuación de 0 (ausente); J) Puntuación de 3 (> 10 células con picnosis). Apoptosis: K) Puntuación de 0 (ausente); L) Puntuación de 3 (> 10 cuerpos apoptóticos). Necrosis: M) Puntuación de 0 (ausente); N) Puntuación de 3 (> 70 \%). Hemorragia: 0) Puntuación de 0 (ausente); P) Puntuación de 3 (> 70 \%). Índice de proliferación: Q) Puntuación de 1 (expresión de Ki67 $<10 \%$ ); R) Puntuación de 3 (expresión de Ki67 > $20 \%$ ). Las puntuaciones se agregaron para dar un valor total y predecir el grado del tumor. En el aumento de 40x, la barra corresponde a una escala de 20 um para la celularidad, pleomorfismo nuclear, mitosis, apoptosis y expresión de Ki67. En el aumento de 10x, la barra corresponde a una escala de $50 \mu \mathrm{m}$ para la hiperplasia endotelial, necrosis y hemorragia. Las características histológicas se señalan con una flecha negra.

obtuvo al sumar las puntuaciones de hiperplasia endotelial y hemorragia, en las que también se encontró diferencia significativa $(p=0.0005)$. El valor de la muerte celular se calculó sumando las puntuaciones de necrosis y apoptosis; también se observó una diferencia significativa $(p<0.0001)$ (Figura 2).

Al considerar la tasa de supervivencia a los 36 meses, el seguimiento se realizó solo en 14/17 pacientes con tumor de bajo grado que recibieron tratamiento (uno murió en el posoperatorio y dos se perdieron durante el seguimiento) y en 22/41 pacientes con tumor de alto grado (cinco fallecieron en el posoperatorio, 11 se perdieron del seguimiento y tres rechazaron el tratamiento posquirúrgico de radioterapia y quimioterapia). De estos pacientes, 13/17 pacientes con bajo grado (76.4\%) y 6/41 con gliomas de alto grado (14.6\%) estaban vivos al final del seguimiento de 36 meses. Se observó diferencia significativa entre los grupos en la supervivencia global $(p<0.0001)$.

\section{Discusión}

Debido a las características heterogéneas de los gliomas, su clasificación y estadificación tumoral origina interpretaciones subjetivas y diferencias en el diagnóstico, por ello 20 a $30 \%$ de los casos se reclasifica al revisar el material tumoral de forma independiente. $^{2}$ Aldape et al. reportaron desacuerdo en el diagnóstico en $23 \%$ de 457 casos evaluados -con mayor discordancia en los casos referidos de hospitales comunitarios y menor en los provenientes de hospitales académicos-; consideraron que $16 \%$ de los diagnósticos discordantes eran clínicamente relevantes para el tratamiento y pronóstico. ${ }^{5} \mathrm{Al}$ parecer, algunas variaciones interobservador se deben a cuestiones técnicas simples como no revisar exactamente el mismo material, pero principalmente al uso de términos subjetivos para diferenciar los grados tumorales, por ejemplo "incremento de la celularidad" o "moderado incremento de la celularidad", usados para diferenciar un astrocitoma anaplásico de un glioma grado II. ${ }^{4}$

Aun cuando el uso combinado de los criterios histológicos y moleculares podría mejorar los resultados, ${ }^{1}$ se requieren nuevos enfoques para la clasificación de los tumores debido a la falta de criterios objetivos, cuantitativos y reproducibles para los diagnósticos histológicos. Lo anterior puede ser importante al considerar que las técnicas moleculares son de difícil acceso en países en desarrollo, en los que la tinción de hematoxilina y eosina es el primer paso en el diagnóstico y el método más accesible para la mayoría de los laboratorios de patología.

Por consiguiente, en este trabajo se seleccionaron los aspectos constantes de los gliomas malignos que permitieran diferenciarlos con mayor certeza de los gliomas 
A

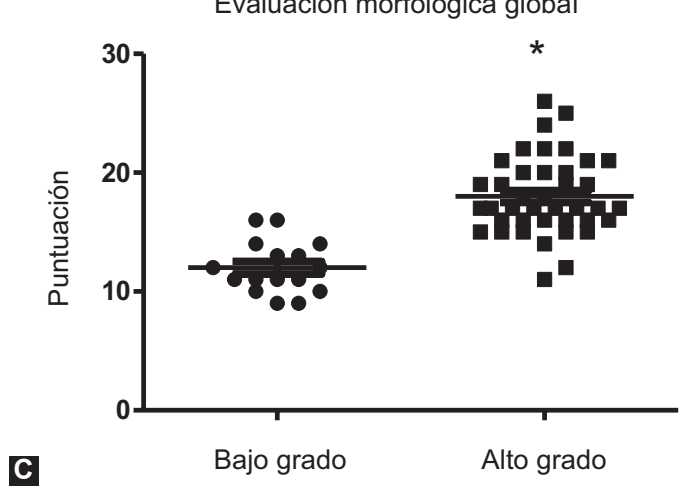

Angiogénesis

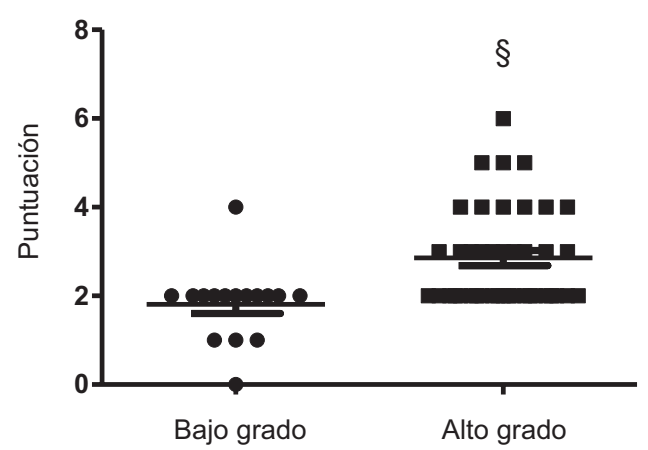

B

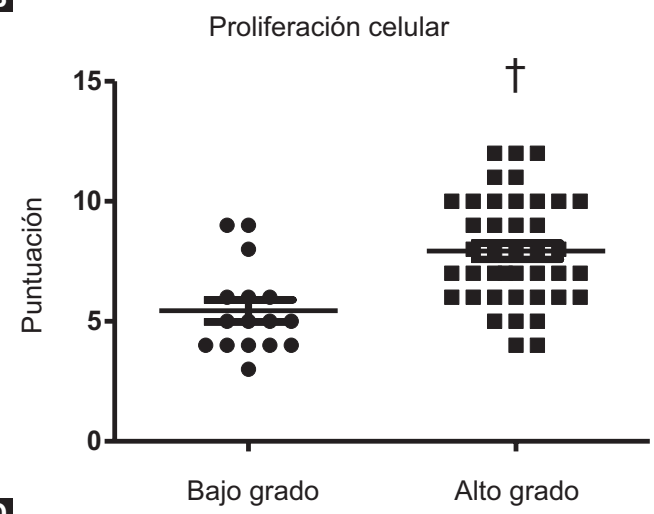

D

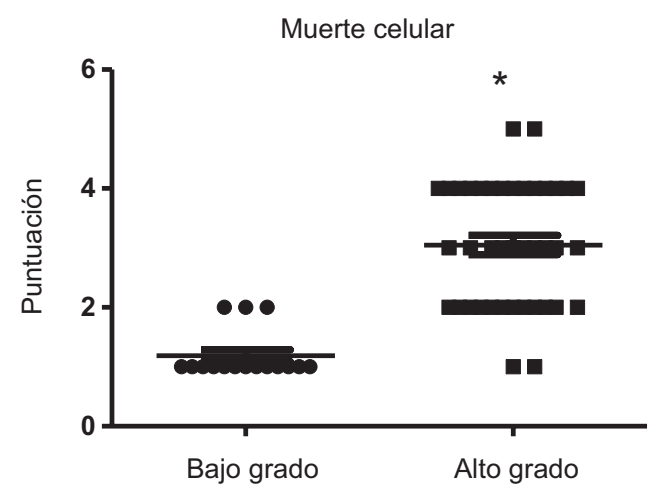

Figura 2. Diferencias en las puntuaciones de los gliomas de bajo y alto grado. A) Evaluación morfológica global (incluye todos los parámetros), ${ }^{*} p<0.0001$. B) Proliferación celular (celularidad + pleomorfismo nuclear + mitosis + Ki67), ${ }^{t} p<0.0002$. C) Angiogénesis (hiperplasia endotelial + hemorragia), ${ }^{s} p<0.0005$; D) Muerte celular (necrosis y apoptosis), ${ }^{*} p<0.0001$.

de bajo grado mediante un análisis objetivo. En primer lugar se consideró la estirpe celular, que puede ser astrocítica, oligodendrocítica o ependimaria, pero también se identificaron casos de "designación no específica". La estirpe astrocítica es la más frecuente en los gliomas de bajo a alto grado (I a IV o glioblastoma). La estirpe oligodendrocítica es la siguiente en frecuencia, pudiendo desarrollar neoplasias hasta grado III. Finalmente, la estirpe menos frecuente es la ependimaria, que también puede desarrollar neoplasias grado III. ${ }^{6}$ En la población analizada predominó la estirpe astrocítica.

En general, dependiendo de su malignidad, los gliomas pueden presentar centro necrótico, proliferación vascular, células gliales neoplásicas, mitosis y pleomorfismo nuclear, con una MF abundante o escasa. Además, también se puede observar la heterogeneidad de las estructuras con apariencia epitelial, variaciones en la morfología celular como células pequeñas, componentes oligoides, células gigantes, gemistocitos, células granulares y células lipidizadas, componentes metaplásicos, proliferación vascular variable y necrosis coagulativa o pseudopaladiza, así como respuesta inmune con linfocitos perivasculares.
Los aspectos histopatológicos de los tumores en crecimiento son la relación entre célula:MF, la presencia de células indiferenciadas y pleomórficas, la identificación de mitosis o células polinucleadas y la expresión de Ki67. En este trabajo, el porcentaje o cantidad de proliferación celular permitió diferenciar entre gliomas de bajo (I y II) y alto grado (III y IV).

En los gliomas de alto grado, las células neoplásicas con diferenciación astrocítica forman prolongaciones citoplásmicas delgadas con apariencia estelar. En contraste, los astrocitomas de bajo grado, como el astrocitoma difuso, generalmente muestran una MF constante, con mayor proporción respecto al número de células. El grado de malignidad aumenta cuando el número de células es mayor que la MF, como en el glioblastoma, de modo que las áreas hipercelulares se pueden encontrar donde la MF es escasa o moderada. ${ }^{7}$ Entre otras alteraciones están las células diferenciadas, células con citoplasma reducido, células con cromatina nuclear compacta o pleomorfismo nuclear de leve a severo. ${ }^{8}$ Las células de glioma pueden estar ligeramente diferenciadas 0 en forma de huso, en el que se puede observar alta actividad 
proliferativa, sin embargo la proliferación es baja cuando hay diferenciación gemistocítica. ${ }^{9}$ Esta característica se asocia con el número de mitosis y es una forma de determinar el grado de malignidad tumoral. El número de mitosis es limitado en gliomas de bajo grado y generalmente es alto en glioblastomas, donde se pueden identificar formas con mitosis típica y atípica. La proliferación celular que se determina a través de la detección inmunohistoquímica de Ki67 es comparable entre los gliomas de alto grado, en los que se observa una expresión entre 15 y $20 \%$ y es significativamente mayor en comparación con los gliomas de grado bajo. La proteína Ki67 participa en la regulación del ciclo celular, por lo tanto, está ausente en las células sin replicación y sus niveles máximos de expresión se alcanzan durante la mitosis. Además, se usa comúnmente como marcador de proliferación celular y se correlaciona positivamente con el grado tumoral y el pronóstico. ${ }^{10}$

La angiogénesis es un parámetro que abarca la presencia de vasos normales y aberrantes, hipoxia y hemorragia. En el presente trabajo se observó que las puntuaciones correspondientes a la angiogénesis y las de la proliferación celular fueron significativamente diferentes entre los gliomas de bajo y alto grado.

La hipoxia y la angiogénesis son dos factores relacionados y su presencia es característica en los gliomas de alto grado. La acumulación celular de factor inducido por hipoxia 1alfa (HIF-1 $\alpha$ ) activa los genes regulados por la hipoxia e induce la angiogénesis mediante la expresión del factor de crecimiento endotelial vascular. ${ }^{11}$ En la angiogénesis, los vasos neoplásicos se forman a partir de vasos preexistentes a través de la migración de células neoplásicas. También se ha propuesto que las células madre neoplásicas regulan y contribuyen a la formación de vasos neoplásicos cuando se diferencian en células endoteliales o mediante la secreción de factores como el factor de crecimiento endotelial vascular o el factor de crecimiento derivado del hepatoma. ${ }^{12}$ La angiogénesis promueve la formación de diferentes patrones microvasculares como mechones glomeruloides, brotes vasculares, agregados vasculares, "vasos de guirnaldas", vasos dilatados, entre otros. Inicialmente, los vasos presentes en la periferia de la lesión pueden detectarse mediante estudios de imagen. En el tumor resecado se corrobora la abundancia de vascularización y la presencia de hiperplasia endotelial (formaciones ovales que se asemejan a corpúsculos renales). La hiperplasia endotelial focal es suficiente para diagnosticar y elevar el grado de malignidad..$^{13}$ En este trabajo se observó de forma importante en los gliomas de alto grado.

En cuanto a la muerte celular, la principal característica del glioblastoma es la presencia de necrosis, debido a que permite una diferencia significativa en los valores promedio de los gliomas de bajo y alto grado. Además, la necrosis predice agresividad en la evolución clínica y se asocia con bajas tasas de supervivencia. ${ }^{14}$ La corroboración histopatológica de la necrosis coagulativa en el glioma puede asumir un efecto secundario a la insuficiencia de suministro de oxígeno. Otro tipo de necrosis característica en el glioblastoma es la estructura en forma de serpentina con un centro necrótico y células neoplásicas gliales con apariencia de pseudoempalizada en la periferia. ${ }^{15,16}$ En el centro del tumor se puede identificar tejido necrótico o restos de MF y adyacentes a estas zonas, cuerpos apoptóticos con baja proliferación celular, probablemente debido a la hipoxia de la región central.

Otra causa probable de necrosis es la oclusión vascular por trombos. Este fenómeno ocurre en áreas hipóxicas adyacentes a los vasos afectados y permite la migración celular a la periferia de la zona necrótica. El porcentaje de necrosis es variable de un tumor a otro, alcanzando hasta $80 \%$ del tejido neoplásico. También se han observado diferencias en los glioblastomas primarios y secundarios, ya que los glioblastomas primarios muestran mayor necrosis que los glioblastomas secundarios. ${ }^{17}$

La apoptosis o muerte celular programada tiene características celulares morfológicas distintivas. En este tipo de muerte celular, los núcleos se distinguen por cromatina granular y fragmentada. Estos cambios se inician a través de la unión de receptores de ligando inductor de apoptosis relacionado con el factor de necrosis tumoral, el receptor de muerte 5 y la activación posterior de caspasa 8. La expresión de FAS se ha observado en células que rodean a la pseudopalizada y la expresión FAS-FASL, en células de la periferia del área de necrosis coagulativa, sin embargo, su asociación con procesos apoptóticos todavía está en discusión. ${ }^{18}$

Es importante aclarar que además de los factores histopatológicos, como factores pronósticos también se han establecido aspectos clínicos como edad, déficit neurológico prequirúrgico, tamaño y ubicación del tumor; aspectos quirúrgicos como grado de resección y biomarcadores como IDH1 mut, metilación del promotor MGMT (no metilado) y la codeleción 1p19q.,19-21 Por consiguiente, la puntuación histopatológica, los aspectos clínicos y 
los biomarcadores pueden usarse en conjunto para predecir la evolución y establecer un tratamiento agresivo o conservador del glioma. . $2,23^{2}$

Las limitaciones del presente trabajo estuvieron determinadas por los siguientes aspectos:

- El pequeño número de pacientes, ya que no se incluyó un número suficiente de gliomas de todos los grados, lo que únicamente permitió diferenciarlos en bajo y alto grado.

- El seguimiento limitado de la evolución de la enfermedad, que no hizo posible determinar la supervivencia global en periodos mayores a tres años.

Por otro lado, habrá que probar la escala propuesta en estudios prospectivos que demuestren que la herramienta se correlaciona mejor con la supervivencia libre de progresión tumoral y supervivencia global, lo cual tomará tiempo, requiriéndose incluir un número mayor de pacientes, para sustentar que los criterios son de utilidad. El presente trabajo no incluye los criterios moleculares propuestos por la Organización Mundial de la Salud en 2016, ${ }^{2}$ porque la intención fue fortalecer la evaluación de las características histológicas que sí pueden ser valoradas en países en desarrollo.

Se concluye que la evaluación morfológica semicuantitativa propuesta permite una clasificación histopatológica más objetiva. Aun cuando la graduación histológica sigue siendo un desafío debido a la superposición de las características morfológicas, puede reducir las discrepancias interobservador en el diagnóstico. La herramienta propuesta podría permitir que el patólogo diagnostique y clasifique el glioma con mayor precisión, lo cual podría ser usado por los clínicos como un factor predictor independiente de la progresión del tumor, junto con las pruebas moleculares propuestas por la Organización Mundial de la Salud, cuando sea posible.

\section{Agradecimientos}

Un agradecimiento especial a la doctora Rocío Lorena Arreola Rosales del Departamento de Anatomía Patológica, Hospital de Especialidades, Centro Médico Nacional Siglo XXI, por su valiosa ayuda al proporcionar el material histopatológico.

\section{Conflictos de intereses}

Los autores declaran no tener conflictos de intereses.

\section{Financiamiento}

Este trabajo fue financiado por el Fondo de Investigación en Salud del Instituto Mexicano del Seguro Social, FIS/IMSS/PRIO/10/012.

\section{Bibliografía}

1. Louis DN, Perry A, Reifenberger G, Von Deimling A, Figarella-Branger D, Cavenee WK, Ohgaki H, et al. The 2016 World Health Organization Classification of Tumors of the Central Nervous System: a summary. Acta Neuropathol. 2016;131:803-820. DOI: 10.1007/s00401-016-1545-1

2. Van den Bent MJ. Interobserver variation of the histopathological diagnosis in clinical trials on glioma: a clinician's perspective. Acta Neuropathol. 2010;120:297-304.

3. Weller M, Wick W, Aldape K, Brada M, Berger M, Pfister SM, et al. Glioma. Nat Rev Dis Primers. 2015:1:15017.

4. Louis DN, Ohgaki H, Wiestler OD, Cavenee WK. WHO Classification of Tumours of the Central Nervous System. Francia: International Agency for Research on Cancer; 2007.

5. Aldape K, Simmons ML, Davis RL, Miike R, Wiencke J, Barger G, et al. Discrepancies in diagnoses of neuroepithelial neoplasms. Cancer. 2000;88:2342-2349.

6. Ostrom QT, Gittleman H, Fulop J, Liu M, Blanda R, Kromer C, et al. CBTRUS Statistical Report: primary brain and central nervous system tumors diagnosed in the United States in 2008-2012. Neuro Oncol. 2015;17:iv1-iv62.

7. Burger PC, Vollmer RT. Histologic factors of prognostic significance in the glioblastoma multiforme. Cancer. 1980;46:1179-1186.

8. Sharma S, Deb P. Intraoperative neurocytology of primary central nervous system neoplasia: a simplified and practical diagnostic approach. J Cytol. 2011;28:147-158.

9. Watanabe K, Tachibana O, Yonekawa Y, Kleiuhes P, Ohgaki H. Role of gemistocytes in astrocytoma progression. Lab Invest. 1997;76:277-284.

10. Thotakura M, Tirumalasetti N, Krishna R. Role of Ki-67 labeling index as an adjunct to the histopathological diagnosis and grading of astrocytomas. J Cancer Res Ther. 2014;10:641-645.

11. Acker T, Plate KH. Hypoxia and hypoxia inducible factors (HIF) as important regulators of tumor physiology. Cancer Treat Res. 2004;117:219-248.

12. Jhaveri N, Chen TC, Hofman FM. Tumor vasculature and glioma stem cells: contributions to glioma progression. Cancer Lett. 2016;380:545-551.

13. Chen L, Lin ZX, Lin GS, Zhou CF, Chen YP, Wang XF, et al. Classification of microvascular patterns via cluster analysis reveals their prognostic significance in glioblastoma. Hum Pathol. 2015;46:120-128.

14. Burger PC, Green SB. Patient age, histologic features, and length of survival in patients with glioblastoma multiforme. Cancer. 1987;59:1617-1625.

15. Brat DJ, Van Meir EG. Vaso-occlusive and prothrombotic mechanisms associated with tumor hypoxia, necrosis, and accelerated growth in glioblastoma. Lab Invest. 2004;84:397-405.

16. Rong Y, Durden DL, Van Meir EG, Brat DJ. "Pseudopalisading" necrosis in glioblastoma: a familiar morphologic feature that links vascular pathology, hypoxia, and angiogenesis. J Neuropathol Exp Neurol. 2006;65:529-539.

17. Ohgaki $H$, Kleihues $P$. The definition of primary and secondary glioblastoma. Clin Cancer Res. 2013;19:764-772.

18. Hao C, Beguinot F, Condorelli G, Trencia A, Van Meir EG, Yong VW, et al. Induction and intracellular regulation of tumor necrosis factor-related apoptosis-inducing ligand (TRAIL) mediated apoptosis in human malignant glioma cells. Cancer Res. 2001;61:1162-1170.

19. Pignatti F, Van den-Bent M, Curran D, Debruyne C, Sylvester R, Therasse $P$, et al. Prognostic factors for survival in adult patients with cerebral low-grade glioma. J Clin Oncol. 2002;20:2076-2084.

20. Stupp R, Brada M, Van den-Bent MJ, Tonn JC, Pentheroudakis G, ESMO Guidelines Working Group. High-grade glioma: ESMO Clinical Practice Guidelines for diagnosis, treatment and follow-up. Ann Oncol. 2014;25:iii93-iii101.

21. Adeberg S, Bostel T, König L, Welzel T, Debus J, Combs SE. A comparison of long-term survivors and short-term survivors with glioblastoma, subventricular zone involvement: a predictive factor for survival? Radiat Oncol. 2014;9:95.

22. Towner RA, Jensen $R L$, Colman $H$, Vaillant $B$, Smith $N$, Casteel $R$, et al. ELTD1, a potential new biomarker for gliomas. Neurosurgery. 2013;72:77-90.

23. Su J, Guo B, Zhang T, Wang K, Li X, Liang G. Stanniocalcin-1, a new biomarker of glioma progression, is associated with prognosis of patients. Tumour Biol. 2015;36:6333-6339. 